\title{
Space Academy: A Journey from Hospital to Mars
}

\author{
Rachael Dixon \\ Edinburgh Children's Hospital Charity \\ Edinburgh, Scotland \\ rachael.dixon@echcharity.org
}

\begin{abstract}
Being in hospital can be a very frightening and lonely experience, especially as a child and especially when in an isolation unit. A patient may be in isolation either because they are infectious to other patients or because they are immunosuppressed, and it is dangerous for them to be around other patients and illnesses. Edinburgh Children's Hospital Charity (ECHC) is developing a Space Academy programme which aims to take the isolation experienced by these children and mirror it with the experience of an astronaut in space, thereby using the idea of space exploration to inspire children who are at their most vulnerable, and hopefully lessen the negative impact hospital experiences may have on a child. ECHC's Space Academy is a 3-week programme which sees each child through the stages of their mission to Mars, right through from pre-launch to debrief. A video-guided app is being developed to take them through their journey, with Tim Peake having agreed to record the introductory video. Each day offers different activities which follow this narrative, and which have been developed to abide by infection control restrictions within the hospital environment. Many of these activities are designed to parallel the work of scientists and astronauts, having used ESA resources aimed at school teachers as a guideline. The programme will therefore provide the children and young people with a way to engage with science whilst in hospital, at a time when they may be missing out on mainstream education. The programme is due to be trialled in the oncology ward of the Edinburgh Royal Hospital for Sick Children in 2020, since many cancer treatments result in a lowered immune system which requires the patients to be in isolation. This paper outlines the need for such a programme, the details of the planned pilot initiative and discusses the potential future development and reach of the project.
\end{abstract}

Keywords—space, Mars, clinical isolation, hospital, STEM, STEAM

\section{INTRODUCTION}

Clinical, or 'source', isolation refers to the increased infection control measures that are put in place when a patient is highly contagious to others, or when they are immunosuppressed and therefore highly susceptible to infection. In such cases, each patient resides in a 1-person isolation cubicle to minimise the spread of infection. Although isolation is necessary for many patients and may be considered beneficial for the quiet and privacy provided, there is much evidence to suggest that clinical isolation results in higher levels of stress, anxiety and depression for patients [1]. This is almost certainly a consequence of the feelings of abandonment, loneliness, neglect and boredom that these patients experience $[1,2]$. This, together with their lowered self-esteem and sense of control, affects these individuals' ability to cope with their situation [3].

Very little research has been done into the impact of clinical isolation on the mental health of children and young people, and how this impact may be lessened. Due to the strict infection control regulations that are in place to minimise the bacteria that can enter and leave each cubicle, it is especially difficult to cater to the non-clinical needs of these children and young people. All things entering the room need to be brand new or be able to be cleaned using disinfectant wipes. Soft toys, board games and arts materials are all seen as bacterial breeding-grounds by clinical staff. It is also required that any staff entering the room first needs to don a plastic apron and thoroughly wash their hands. Therefore, there is an increased need for creativity when trying to meet these non-clinical needs.

Play specialists at the Royal Hospital for Sick Children (RHSC) in Edinburgh consulted patients who have had experience of clinical isolation for ideas on how the experience could be improved. One of the children likened being in isolation to being an astronaut, and the idea took off from there. Staff at Edinburgh Children's Hospital Charity (ECHC) have put together 'Space Academy' - a programme of activities which aims to combat the social isolation, lack of control and boredom that these children and young people experience, whilst abiding by infection control regulations, with the overarching theme of space travel. The project uses STEAMbased activities in order to help participants feel part of a wider project and community, allow them to engage with fun, educational material and provide them with the ability to transform their forced environment.

This paper explores the potential role that STEM education, together with artistic expression, could have in combating the negative effects of clinical isolation. An outline is provided of the motivation behind the project, the structure of the 3-week programme which is due to be trialled in the RHSC in 2020, and the future development and potential of the project.

\section{From Hospital to MARS}

Upon consulting Romain Charles, an ESA member of the 520-day isolation crew of the Mars500 mission, it became clear just how many parallels could be made between the life of an astronaut and the lives of the children and young people in clinical isolation. The regimented schedule that members followed during Mars500, which is of a similar structure to that of ISS astronauts, is comparable to the routine that hospital patients follow. According to $\mathrm{Mr}$. Charles, an average day on Mars500 would follow a schedule similar to: 
- 08:00 - Medical checks (weight, blood pressure, ECG, etc.)

- $\quad 08: 30$ - Breakfast

- 09:00 - Work (experiments)

- 12:30- Lunch

- 13:30 - Physical exercise

- 14:00 - Complete experiments from earlier in the day if necessary, read/write emails to friends and family, improve Russian

- 18:00 - Dinner

- 19:00 onwards - Common activities e.g. games console, tv

The routine medical tests, strict meal times (with a limited, repetitive menu) and restricted contact with friends and family are all aspects of daily life in clinical isolation. Though these similarities are at the core of the Space Academy project, it was necessary to bear in mind one important difference throughout its development - astronauts choose to go into space and are able to physically and mentally prepare themselves for it; these children and young people do not choose to be in hospital.

From there, it became about developing a mission plan - a story that had tangible goals that could be achieved by participants within a relatively short space of time, and that would allow for a coherent range of space-themed STEM topics to be explored. A journey to Mars seemed the obvious choice due to the presence of Mars exploration ambitions in the media currently which provided a lot of scope for potential activities. The 'astronauts' (participants) start their journey with a Pre-Launch introduction to the programme. They then go through Launch, a 'visit' to the International Space Station, Life in Space and Mars before their Return to Earth, all with the overarching mission of Mars exploration. At its core, the journey makes a parallel to the experience of a patient - being in an unfamiliar setting surrounded by alien technology until the hopeful return home. However, due to the unfortunate fact that not all participants will reach this part of their own journey, this theme has only a subtle presence.

The storyline of a journey to Mars also allows for STEAM subjects to be explored in an unconventional way which aims to capture the imagination of participants. The patients in isolation are often there for extended periods of time and, as such, miss out on large portions of their schooling. At the RHSC in Edinburgh, patients expected to be in hospital for more than five nights are automatically offered teaching (which they can choose to engage with depending on how they are feeling each day). However, there are only two teachers for a hospital with roughly 100 bed spaces. This, together with the additional restrictions associated with teaching children and young people in isolation, both limits the time spent learning and the subjects that they are able to engage with, since English and maths are prioritised by NHS teaching staff. Therefore, a programme which allows patients to engage with educational STEM material, in a way which encourages creativity, would be an innovative resource for combating the immense boredom that these patients face.

Each phase of the journey includes activities which relate to that phase. Several of these activities encourage participants to transform their cubicle into a space-themed haven, therefore giving them the ability to impact their forced environment. Research suggests that the best way of minimising the negative impact clinical isolation can have is to provide patients with a sense of control [3].

Though the activities are mainly designed to be enjoyable, there is also an opportunity for self-led learning. It was decided that a programme consisting of short daily activities over 3 weeks would be of suitable length to achieve tangible goals whilst remaining interesting for participants. The programme is designed to be flexible to the child/young person so that they are able to pick it up and put it down depending on when they feel up to it, and so they can tailor the experience to what interests them. None of the activities are compulsory and participants do not have to stick to the proposed structure. There is also the option during each activity to go into varying levels of theoretical detail depending on the age and interests of the child/young person.

\section{The Space Academy Programme}

The 3-week pilot programme that has been devised by ECHC follows a 'journey to Mars'. It is due to be trialled in the oncology and haematology ward of the RHSC in 2020 since many of the patients in this ward become immunosuppressed due to their treatment and therefore are required to be in clinical isolation.

An app and logbook are being developed to accompany the programme which will allow participants to reflect upon their experiences. The app will guide the participants through each day of their journey, with an introductory video from Tim Peake to brief the 'astronauts' of their mission. The app will include instructions for each of the daily activities, fun facts and information about their 'crew' (doctors, nurses and play specialists). Each participant will have their own digital tablet whilst in hospital which will allow access to the app and provide them with a platform to complete certain activities.

Pre-Launch will include the welcome from Tim Peake and will aim to prepare the participants for their journey into space. The participants will get the chance to add to their own profile which will include their likes and dislikes, their mission badge (that they will have the opportunity to design) and their very own flight playlist (like ISS astronauts have for their launch days). Other pre-launch topics and activities include spacecraft materials testing, a captain's checklist, rocket design and 3D printing, and aerodynamics.

After lessons on how to count down in various languages (to emphasise the presence of many nationalities aboard the ISS), a video will play on the app which shows lift off from the RHSC, hence concluding the Launch phase of the mission.

The participants will then find themselves aboard the International Space Station, where they will complete activities to further understand life on Earth and in space. These 
activities will involve experimenting with gravity, delayed communications with Earth, and a robotic arm. Aboard the ISS, participants will have their first opportunity to feed back to 'Mission Control' (their school). This offers participants the chance to engage with their peers and feel part of a wider project and community, whilst also providing their peers an insight into, and understanding of, their hospital experience. It is hoped that this will lessen the extent of their social isolation and make the transition back into school a less daunting experience. Many of the activities completed during the ISS phase of the mission were adapted from ESA's ISS Education Kit for teachers.

The journey from the ISS to Mars is encapsulated in the Life in Space section. The participants will learn all about their solar system and of the constellations that can be seen from within it. This will involve learning about the Sun, and the electromagnetic radiation that it emits.

Once on Mars, participants are challenged to build a rover to help investigate whether life has ever been sustained on Mars. ECHC are working in collaboration with Airbus, Centre for Life, ESA, ESERO, and UKSA to develop a Mars Challenge which is envisaged to engage 50,000 people in space educational activities. As such, the Mars section of ECHC's Space Academy will consist of activities and materials designed for the wider project, making the necessary adaptations for use in the hospital environment. During this phase of the mission, participants are encouraged to consider what would be needed to sustain a civilisation on Mars, asking questions like: should there be a monarchy on Mars? If so, who would be on the throne?

Upon arrival back to Earth, the participants will be debriefed on their mission to Mars and will be encouraged to reflect on the contents of their logbook. All participants of Space Academy will be awarded a personalised certificate, regardless of whether they have completed one day or twentyone days of the programme. The reality of the hospital environment may mean that few participants actually complete their Space Academy journey - whether that be due to early discharge, or constraints set by how well or unwell they may feel at the time - which is why it is important to reinforce their achievement no matter what stage has been reached. The app will also award participants with digital badges when activities and sections that are completed to reinforce this sense of achievement.

\section{TESTING}

With two or three activities having been assigned to each day, it was important to ensure the activities are fun, engaging and accessible to multiple age groups during development. Meeting these criteria is made all the more difficult by the strict infection control regulations within the hospital. Several of the activities have been trialled around the wards of the RHSC, as well as at ECHC's youth group for 12-18 year-olds, to ensure these criteria have been met. The response from patients, staff and parents/carers to the activities was very positive. One patient in particular vocalised the benefit of such activities for children and young people that miss out on large amounts of mainstream education as a result of being in hospital. The young person was very interested in the theory behind the activities, since he had missed out on the science that would have been covered in school whilst undergoing numerous rounds of chemotherapy. That said, the same activities were also enjoyed by many others, including two siblings (aged 4 and 6 years old) who were in the hospital visiting their sister.

Most activities were accessible to the suitable age range, since it was ensured that the underlying science/theory was not key to being able to engage with the activity. That way, the children and young people participating in these activities could take as much as they wanted from the experience. Trialling the activities in this way ensured that the programme and app were developed in such a way as to ensure participants can engage with varying levels of information and therefore reinforce that the programme is meeting the needs of children and young people of various ages.

\section{COnClusion and Future Development}

It is evident from research and recorded patient experiences at the RHSC in Edinburgh that there is a need to better accommodate the non-medical needs of children and young people admitted to clinical isolation. Staff at ECHC have developed the Space Academy programme in hope of achieving this through using STEM-based activities - in an unconventional, creative way - to lessen the negative impact that clinical isolation can have.

Trialling the project in the oncology and haematology ward of the RHSC will allow for any teething problems to be ironed out, and feedback to be gathered from participants. After such a time, the programme will be made available to all children and young people admitted to clinical isolation at the RHSC. Should other hospitals then show interest in the project, it would be reasonably simple to share the materials since the only part of the programme specific to Edinburgh is a short video clip where the participants 'take off' from Earth and leave the RHSC.

More than 150 children and young people are admitted to clinical isolation in Edinburgh alone. Space Academy is the first project of its kind which attempts to improve this experience.

\section{ACKNOWLEDGMENTS}

I would like to thank Airbus, Centre for Life, ESA, ESERO and UKSA for their contributions to this project. I would also like to thank the School of Physics and Astronomy at the University of Edinburgh for making my presence at SSEA 2019 possible.

\section{REFERENCES}

[1] R.L. Barratt, R. Shaban, and W.Moyle, "Patient experience of source isolation: lessons for clinical practice," Contemporary Nurse, vol. 39, pp. 180-193, June 2011.

[2] L.Saiman et al., "Infection prevention and control guideline for cystic fibrosis: 2013 update," Infection Control and Hospital Epidemiology, vol. 35, pp. S1-S67, August 2014.

[3] J. Gammon, "Analysis of the stressful effects of hospitalisation and source isolation on coping and psychological constructs," International Journal of Nursing Practice, vol. 4, pp. 84-96, June 1998. 